\title{
Indirect Treatment Comparison of Nivolumab Versus Observation or Ipilimumab as Adjuvant Therapy in Resected Melanoma Using Pooled Clinical Trial Data
}

\author{
Morganna Freeman - Keith A. Betts · Shan Jiang · Ella X. Du • \\ Komal Gupte-Singh · Yichen Lu · Sumati Rao · Alexander N. Shoushtari
}

Received: June 19, 2019 / Published online: August 22, 2019

(C) The Author(s) 2019

\section{ABSTRACT}

Introduction: Nivolumab has been approved in patients with melanoma with lymph node involvement or metastatic disease who have undergone complete resection, in the adjuvant setting. A pivotal trial compared nivolumab with ipilimumab; however, no head-to-head trial exists comparing nivolumab to observation, a common comparator in the adjuvant setting. Here, we compared the efficacy and cost-effectiveness of nivolumab with

Enhanced Digital Features To view enhanced digital features for this article go to https://doi.org/10.6084/ m9.figshare.9327251.

Electronic Supplementary Material The online version of this article (https://doi.org/10.1007/s12325019-01060-y) contains supplementary material, which is available to authorized users.

M. Freeman $(\varangle)$

City of Hope Comprehensive Cancer Center, Los Angeles, CA, USA

e-mail: mofreeman@coh.org

K. A. Betts - E. X. Du · Y. Lu

Analysis Group, Inc., Los Angeles, CA, USA

S. Jiang · K. Gupte-Singh · S. Rao

Bristol-Myers Squibb, Princeton, NJ, USA

A. N. Shoushtari

Memorial Sloan Kettering Cancer Center, New York, NY, USA observation or ipilimumab as adjuvant therapies in resected stage IIIB/C melanoma.

Methods: Patient data were pooled from the EORTC 18071 and CheckMate 238 trials using propensity score weighting and adjusting for cross-trial differences. Number needed to treat (NNT) and costs per recurrence-free life-month (RFLM) at 12, 16, 18, and 24 months (as data allowed) were estimated. Costs included drug acquisition, administration costs, and direct medical costs. Sensitivity analyses including patients with stage IIIB/C and resected stage IV melanoma were conducted.

Results: A total of 1287 patients (278 nivolumab, 365 observation, and 644 ipilimumab) with resected stage IIIB/C melanoma were pooled. NNTs to achieve one additional recurrence-free survivor with nivolumab versus observation were 3.93 at 12 months and 3.42 at 24 months; NNTs for nivolumab versus ipilimumab were 7.97 at 12 months and 6.43 at 24 months. Mean drug costs per RFLM were lower for nivolumab at 12,18 , and 24 months, respectively (nivolumab: \$13,447, \$9462, and $\$ 7370$; ipilimumab: $\$ 52,734, \$ 40,484$, and $\$ 33,875)$. Mean medical costs per RFLM were the lowest for nivolumab versus observation or ipilimumab at 12 months ( $\$ 449$ versus $\$ 674$ or $\$ 1531$ ) and 16 months ( $\$ 383$ versus $\$ 808$ or \$1316). The sensitivity analysis results were consistent with the base case.

Conclusion: For resected melanoma, adjuvant nivolumab is both clinically effective and cost- 
effective compared with observation or ipilimumab. Adjuvant nivolumab was associated with a lower drug cost per RFLM compared with ipilimumab, and a lower medical cost compared with observation. Future analyses incorporating long-term follow-up data may help increase understanding of the economic impact of nivolumab in the adjuvant setting.

Funding: Bristol-Myers Squibb Company.

Keywords: Adjuvant treatment; Costs per recurrence-free life-month; Costs per recurrence-free survivor; Ipilimumab; Melanoma; Nivolumab; Number needed to treat; Observation

\section{INTRODUCTION}

Melanoma is the most aggressive and deadliest form of skin cancer [1]. Over the past decade, the incidence of melanoma has steadily increased in the USA, growing at an average yearly rate of $1.5 \%$ [2]. In 2019, an estimated 96,480 Americans are likely to be newly diagnosed with invasive melanoma and 7230 deaths are predicted [3]. The 5-year survival rate for localized melanoma is $99 \%$, decreases to $63 \%$ with lymph node involvement, and declines further to $20 \%$ when metastases reach distant sites or organs [4], most commonly the lungs, bones, liver, and brain [5].

Treatment largely depends on the primary stage and nodal involvement, if present, of melanoma [6]. The mainstay of treatment for early-stage (IA-IIA) and locally advanced melanoma (IIB/C) is wide surgical excision of the primary tumor, with lymphoscintigraphy and nodal sampling in cases of confirmed spread to regional lymph nodes [7-9]. Although treatment in the earlier disease stages can be curative, patients who have primary melanoma with increased tumor thickness, ulceration, increased mitotic rate, or regional nodal metastases are at a higher risk for recurrence $[6,10]$, with particular attention to nodal involvement for upstaging of disease. In one US study, the 5-year risk of melanoma recurrence at any site was estimated at $48 \%$ for stage IIIA, $71 \%$ for stage IIIB, and $85 \%$ for stage IIIC, based on the American Joint Committee on Cancer (AJCC) 7th edition [11].

Recently, new treatment options, including immune checkpoint inhibitors and targeted therapies, have been added to the adjuvant treatment armamentarium for resected melanoma owing to their clinical benefit in improving recurrence-free survival (RFS) [12-15]. The most recent National Comprehensive Cancer Network (NCCN) guidelines recommend observation or adjuvant therapyincluding nivolumab, pembrolizumab, or dabrafenib plus trametinib (for patients with the $B R A F$ V600 mutation)-to prevent recurrence following tumor resection [15].

Although no longer recommended in the most recent NCCN guidelines, ipilimumab was the first immune checkpoint inhibitor approved in the USA as adjuvant therapy for stage III melanoma [12]. In the phase 3 EORTC 18071 trial of patients with resected stage IIIA-C melanoma [16, 17], ipilimumab $10 \mathrm{mg} / \mathrm{kg}$ was associated with significantly improved median RFS (2.7 versus 1.5 years; $P<0.001$ ) and 7 -year overall survival (OS) rate $(60.0 \%$ versus $51.3 \%$; $P=0.0021)$ compared with observation [18]. Despite demonstrated clinical efficacy, the use of ipilimumab as adjuvant treatment for stage III melanoma has been limited by its cost and considerable toxicities $[12,19]$.

Nivolumab, an immune checkpoint inhibitor that targets the programmed cell death 1 protein (PD-1), was approved in December 2017 in the USA for the adjuvant treatment of patients with melanoma with lymph node involvement or in patients with metastatic disease who have undergone complete resection [20]. In the phase 3 CheckMate 238 trial, nivolumab exhibited a significantly higher RFS rate (62.6\% versus $50.2 \% ; P<0.001)$ at 24 months [21] and a lower rate of grade $3 / 4$ treatmentrelated adverse events (14.4\% versus $45.9 \%)$ at 18 months [22] compared with ipilimumab when used as adjuvant treatment for resected stage III and IV melanoma.

A limitation of the CheckMate 238 trial was that nivolumab was not trial-compared with observation/placebo, and cross-trial comparisons of currently approved therapies are limited by trial design and patient populations. 
More importantly, in an era of value-based decision-making in oncology, the cost-effectiveness of these therapies in the adjuvant setting is an overlooked area of investigation. This study assessed the efficacy and cost-effectiveness of adjuvant nivolumab compared with observation or adjuvant ipilimumab by pooling data from two clinical trials, EORTC 18071 (comparing ipilimumab versus observation [16]) and CheckMate 238 (comparing nivolumab versus ipilimumab [22]), in terms of number needed to treat (NNT) and costs per recurrencefree life-month and costs per recurrence-free survivor. The aims of this study are to understand the comparative efficacy and cost-effectiveness of nivolumab versus observation or ipilimumab in the adjuvant setting among patients with a high risk of melanoma recurrence after surgical resection of the primary tumor and provide valuable information to patients, payers, clinicians, and other healthcare decision-makers.

\section{METHODS}

\section{Data Source}

Individual patient-level data were pooled from two clinical trials, EORTC 18071 [16] and CheckMate 238 [22]. Both trials were phase 3, randomized, double-blind studies with RFS as a primary endpoint and OS as a secondary endpoint.

EORTC 18071 [16] compared ipilimumab with observation after complete resection of stage IIIA-C cutaneous melanoma. Patients received either ipilimumab $(10 \mathrm{mg} / \mathrm{kg})$ or observation every 3 weeks for four doses and then every 12 weeks for up to 3 years. The median follow-up of the currently available EORTC 18071 trial data was 6.9 years [18]. CheckMate 238 [22] compared nivolumab with ipilimumab as adjuvant therapy after complete resection of stage IIIB/C or IV (AJCC 7th edition) melanoma. Patients received either nivolumab (3 mg/kg every 2 weeks) or ipilimumab $(10 \mathrm{mg} / \mathrm{kg}$ every 3 weeks for four doses and then every 12 weeks) for up to 1 year. The minimum follow-up time of currently available
CheckMate 238 trial data is 24 months [21]. In this study, clinical trial data within 24 months from both trials were used. Original data from the EORTC 18071 and the CheckMate 238 trials were collected within an ethical framework. These clinical trials were performed in accordance with the Declaration of Helsinki, had approval from respective institutional review boards, and had obtained informed consent from all participants $[16,22]$.

\section{Study Population and Propensity Score Weighting}

Patients with stage IIIB/C cutaneous melanoma were included in this study as they were the common population included in each of the individual clinical trials. Data from the EORTC 18071 [16] and CheckMate 238 [22] trials were pooled together using propensity score weighting, adjusting for cross-trial differences in age, sex, race, Eastern Cooperative Oncology Group (ECOG) performance status, time from resection to randomization, disease stage, tumor ulceration status, lymph node involvement (microscopic/macroscopic), lactate dehydrogenase (LDH) levels, and presence of pretreatment events (e.g., lymphedema, peripheral edema, increased alanine aminotransferase, fatigue). Patients with any missing baseline characteristics included in the propensity score weighting were removed. After adjustment, baseline characteristics were balanced between the two trials. The Hosmer-Lemeshow test was used to assess the goodness of fit. Baseline characteristics before/after weighting were compared using chi-square tests/weighted chi-square tests for categorical variables and analysis of variance (ANOVA)/weighted ANOVA for continuous variables, respectively.

\section{NNT per Additional Recurrence-Free Survivor}

Using the pooled patient data, we calculated the NNT per additional recurrence-free survivor as the reciprocal of the absolute risk reduction [23] (the absolute difference between the RFS rates of nivolumab versus ipilimumab and of 
nivolumab versus observation) at 12,18 , and 24 months. The corresponding 95\% confidence intervals (CIs) were calculated on the basis of the approach described by Altman and Andersen [23].

\section{Costs per Recurrence-Free Life-Month and Recurrence-Free Survivor}

Using the pooled patient data, we calculated costs per recurrence-free life-month as the costs per patient divided by the area under the Kaplan-Meier RFS curve for each treatment. Costs per recurrence-free survivor were calculated as costs per patient divided by the RFS rate for each treatment. Both the costs per recurrence-free life-month and per recurrence-free survivor were evaluated separately for drug acquisition costs, drug administration costs, medical costs, and total costs, throughout different periods of follow-up, as detailed below.

\section{Drug Acquisition and Administration Costs}

Drug acquisition costs per patient were calculated as the product of the wholesale acquisition costs unit price as of January 5, 2018 [24] (Supplementary Table S1) and the total number of doses received for each treatment during each follow-up period $(12,16,18$, and 24 months). Drug administration costs per patient were calculated as the product of the unit costs per chemotherapy intravenous infusion-obtained from the Medicare physician fee schedule based on Healthcare Common Procedure Coding System codes [25] (Supplementary Table S1)-and the total number of doses received for each treatment during each followup period $(12,16,18$, and 24 months).

\section{Medical Costs}

Medical costs per patient were calculated as the sum of the products of the unit costs [26] of four types of medical visits (hospitalization day, emergency department visit, outpatient visit, and home healthcare) and the total number of each type of medical visit, as reported in the two trials. Medical visits were reported only up to 128 days after the last dose or the discontinuation date (i.e., up to 16 months) in the
CheckMate 238 trial. As such, because medical costs calculated at 18 and 24 months may not fully capture the medical visits that occurred after 16 months, they were not included in this study.

\section{Total Costs}

Total costs per patient were calculated as the sum of drug acquisition costs, drug administration costs, and medical costs per patient through months 12 and 16 (given that no medical costs were available at 18 and 24 months). All costs were inflated to 2018 US dollars.

\section{Sensitivity Analysis}

In the primary analysis described above, patients from the CheckMate 238 study were excluded if they had stage IV disease, to be consistent with the design of the EORTC 18071 study. As a sensitivity analysis, the NNT, costs per recurrence-free life-month, and costs per recurrence-free survivor of nivolumab versus ipilimumab were assessed among patients with stage IIIB/C or IV melanoma only using data from the CheckMate 238 trial. The sensitivity analyses were used to validate the results of nivolumab versus ipilimumab obtained in the primary analysis based on the pooled clinical trial data.

\section{RESULTS}

\section{Patient Population and Baseline Characteristics}

A total of 1287 patients with stage IIIB/C cutaneous melanoma were pooled from the two trials, 725 from EORTC 18071 and 562 patients from CheckMate 238; the patient selection process is detailed in Fig. 1 . Of the 1287 patients, 278 received nivolumab, 365 received placebo/observation, and 644 received ipilimumab. After propensity score weighting, the baseline characteristics of the patients were similar between trials (Table 1 ). The mean age was approximately 52 years, $60 \%$ were men, 


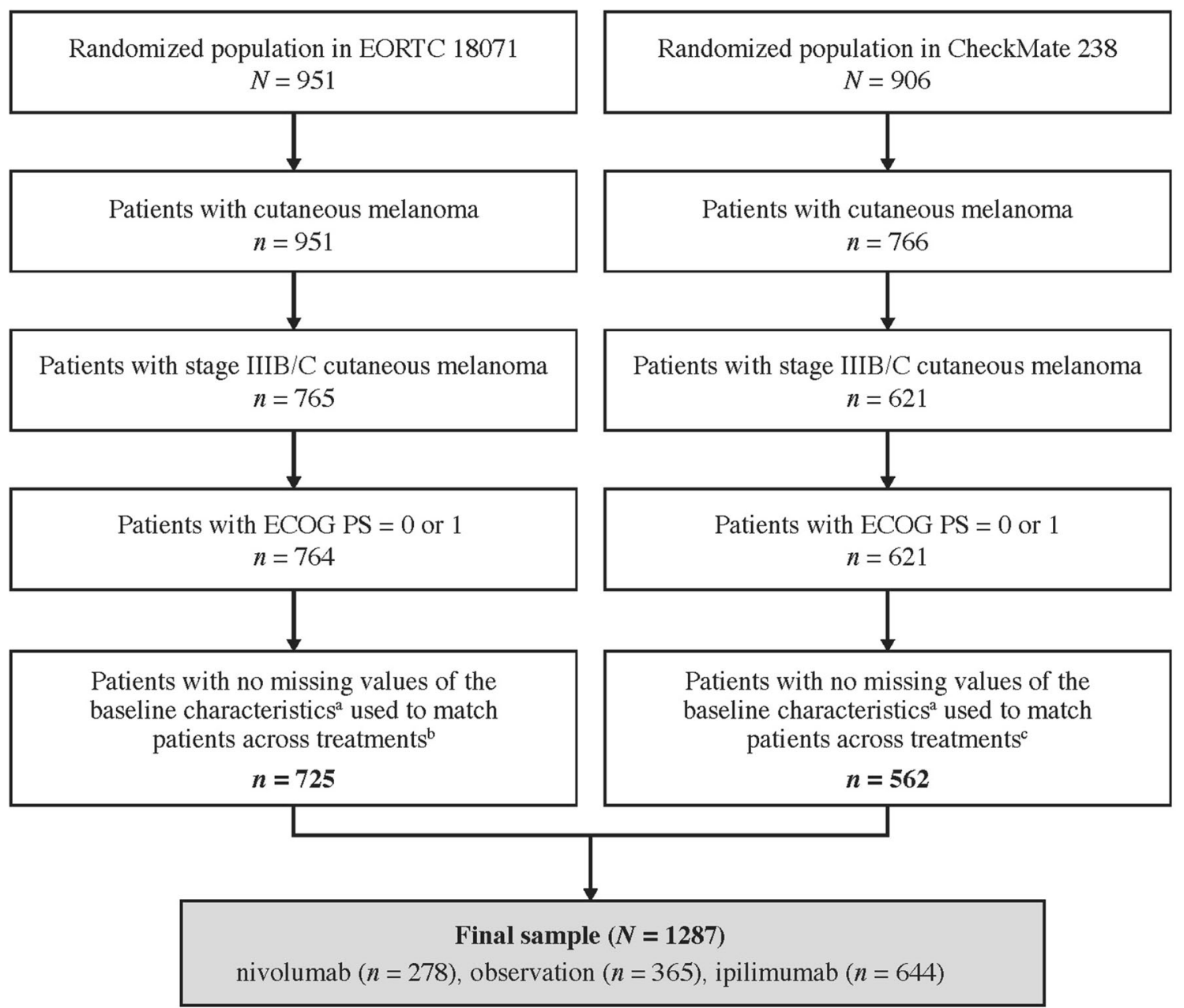

Fig. 1 Selection of the pooled patient population. ECOG PS Eastern Cooperative Oncology Group performance status. ${ }^{a}$ Age, sex, race, ECOG PS, time from resection to randomization, disease stage, tumor ulceration, lymph node involvement (microscopic/macroscopic), lactate dehydrogenase (LDH), and pretreatment events. ${ }^{\mathrm{b}} 39$

approximately $92 \%$ had an ECOG performance status of 0 , a similar proportion of patients had stage IIIB and stage IIIC melanoma, and approximately $68 \%$ and $32 \%$ had macroscopic and microscopic lymph node involvement, respectively. A total of $48.4 \%$ of patients in EORTC 18071 and $50.4 \%$ in CheckMate 238 had tumor ulceration $(P=0.53)$. The $P$ value for the Hosmer-Lemeshow goodness of fit test was 0.0665 , suggesting that the model fits the data well. patients were excluded because of missing baseline data: two had missing race and 38 had missing tumor ulceration status. ${ }^{\mathrm{c}} 59$ patients were excluded because of missing baseline data: 16 had missing tumor ulceration status, 38 had missing lymph involvement status, and eight had missing baseline LDH status

\section{NNT per Additional Recurrence-Free Survivor}

After propensity score weighting, the RFS rates for nivolumab, observation, and ipilimumab were $74.1 \%, 48.7 \%$, and $61.6 \%$ at 12 months, respectively; $70.0 \%, 41.8 \%$, and $54.5 \%$ at 18 months, respectively; and $66.8 \%, 37.6 \%$, and $51.3 \%$ at 24 months, respectively (Table 2 ).

At 12 months, the NNT per additional recurrence-free survivor was 3.93 (95\% CI 3.02, 
Table 1 Baseline characteristics before and after weighting

\begin{tabular}{|c|c|c|c|c|c|c|}
\hline & \multicolumn{3}{|c|}{ Before weighting } & \multicolumn{3}{|c|}{ After weighting } \\
\hline & $\begin{array}{l}\text { EORTC } \\
18071, \\
n=725\end{array}$ & $\begin{array}{l}\text { CheckMate } \\
238, \\
n=562\end{array}$ & $P$ value $^{\mathbf{a}}$ & $\begin{array}{l}\text { EORTC } \\
18071, \\
n=725\end{array}$ & $\begin{array}{l}\text { CheckMate } \\
238, \\
n=562\end{array}$ & $P$ value $^{\mathbf{a}}$ \\
\hline Mean age, years $(\mathrm{SD})$ & $51.9(12.9)$ & $53.6(13.6)$ & $<0.05$ & $52.7(12.8)$ & $52.4(13.7)$ & 0.75 \\
\hline \multicolumn{7}{|l|}{ Sex, \% } \\
\hline Female & 36.1 & 42.2 & $<0.05$ & 40.0 & 39.9 & 0.99 \\
\hline Male & 63.9 & 57.8 & & 60.1 & 60.1 & \\
\hline \multicolumn{7}{|l|}{ Race, \% } \\
\hline White & 99.7 & 95.7 & $<0.001$ & 97.4 & 98.0 & 0.74 \\
\hline Other $^{\mathrm{b}}$ & 0.3 & 4.3 & & 2.6 & 2.0 & \\
\hline \multicolumn{7}{|l|}{ ECOG PS, \% } \\
\hline 0 & 93.9 & 90.8 & $<0.05$ & 91.7 & 92.5 & 0.69 \\
\hline 1 & 6.1 & 9.3 & & 8.3 & 7.5 & \\
\hline $\begin{array}{l}\text { Mean time from resection to } \\
\text { randomization, weeks (SD) }\end{array}$ & $9.2(2.2)$ & $9.0(2.8)$ & 0.17 & $9.2(2.3)$ & $9.2(2.7)$ & 0.87 \\
\hline \multicolumn{7}{|l|}{ Disease stage at baseline, $\%^{\mathrm{c}}$} \\
\hline Stage IIIB & 54.2 & 44.8 & $<0.01$ & 50.4 & 51.5 & 0.72 \\
\hline Stage IIIC & 45.8 & 55.2 & & 49.6 & 48.5 & \\
\hline \multicolumn{7}{|l|}{ Tumor ulceration, \% } \\
\hline Absent & 45.0 & 59.3 & $<0.001$ & 51.6 & 49.6 & 0.53 \\
\hline Present & 55.0 & 40.8 & & 48.4 & 50.4 & \\
\hline \multicolumn{7}{|l|}{ Lymph node involvement, \% } \\
\hline Macroscopic & 70.6 & 62.1 & $<0.01$ & 68.2 & 68.3 & 0.98 \\
\hline Microscopic & 29.4 & 37.9 & & 31.8 & 31.8 & \\
\hline \multicolumn{7}{|l|}{ Baseline LDH, \% } \\
\hline$\leq \mathrm{ULN}$ & 96.6 & 93.1 & $<0.01$ & 95.4 & 95.1 & 0.79 \\
\hline$>\mathrm{ULN}$ & 3.5 & 6.9 & & 4.6 & 4.9 & \\
\hline
\end{tabular}


Table 1 continued

\begin{tabular}{|c|c|c|c|c|c|c|}
\hline & \multicolumn{3}{|c|}{ Before weighting } & \multicolumn{3}{|c|}{ After weighting } \\
\hline & $\begin{array}{l}\text { EORTC } \\
18071, \\
n=725\end{array}$ & $\begin{array}{l}\text { CheckMate } \\
238, \\
n=562\end{array}$ & $P$ value $^{\mathrm{a}}$ & $\begin{array}{l}\text { EORTC } \\
18071, \\
n=725\end{array}$ & $\begin{array}{l}\text { CheckMate } \\
238, \\
n=562\end{array}$ & $P$ value ${ }^{a}$ \\
\hline Patients with pretreatment events, $\%^{\mathrm{c}}$ & 20.8 & 18.2 & 0.26 & 18.9 & 19.0 & 0.97 \\
\hline
\end{tabular}

The $P$ value for the Hosmer-Lemeshow goodness of fit test was 0.0665

ECOG PS Eastern Cooperative Oncology Group performance status, $L D H$, lactate dehydrogenase, $S D$ standard deviation, $U L N$ upper limit of normal

a $P$ value $<0.05$ is considered significant

b Other race included Asian (1 in EORTC 18071; 23 in CheckMate 238) and Native Hawaiian or other Pacific Islander (1 each in EORTC 18071 and CheckMate 238)

c In the EORTC 18071 trial, the most frequent pretreatment events were increased alanine aminotransferase $(1.5 \%$ ipilimumab; $1.7 \%$ observation), fatigue (1.3\% ipilimumab; $1.5 \%$ observation), peripheral edema (1.3\% ipilimumab; $0.8 \%$ observation), increased lipase ( $0.4 \%$ ipilimumab; $1.1 \%$ observation), lymphedema ( $0.6 \%$ ipilimumab; $1.3 \%$ observation), and diarrhea ( $0.4 \%$ ipilimumab; $1.1 \%$ observation). In the CheckMate 238 trial, the most frequent pretreatment events were lymphedema $(3.5 \%)$ in the nivolumab group, and lymphedema $(2.6 \%)$ and peripheral edema $(2.6 \%)$ in the ipilimumab group

Table 2 NNT per additional recurrence-free survivor among patients with stage IIIB/C melanoma

\begin{tabular}{|c|c|c|c|c|c|c|}
\hline Data source & $\begin{array}{l}\text { Treatment } \\
\text { vs. } \\
\text { comparator }\end{array}$ & $\begin{array}{l}\text { Time } \\
\text { horizon, } \\
\text { months }\end{array}$ & $\begin{array}{l}\text { RFS rate of } \\
\text { treatment }^{\mathrm{a}} \text {, } \\
\%\end{array}$ & $\begin{array}{l}\text { RFS rate of } \\
\text { comparator }^{\mathrm{a}} \text {, } \\
\%\end{array}$ & $\begin{array}{l}\text { Absolute } \\
\text { risk } \\
\text { reduction, } \\
\%\end{array}$ & $\begin{array}{l}\text { NNT of treatment } \\
\text { vs. comparator } \\
(95 \% \mathrm{CI})\end{array}$ \\
\hline \multirow{3}{*}{$\begin{array}{l}\text { Pooled EORTC } 18071 \\
\text { and CheckMate } 238 \\
\text { (stage IIIB/C) }^{\mathrm{b}}\end{array}$} & Nivolumab & 12 & 74.1 & 48.7 & 25.5 & $3.93(3.02,5.61)$ \\
\hline & \multirow{2}{*}{$\begin{array}{l}\text { vs. } \\
\text { observation }\end{array}$} & 18 & 70.0 & 41.8 & 28.2 & $3.55(2.78,4.91)$ \\
\hline & & 24 & 66.8 & 37.6 & 29.3 & $3.42(2.69,4.67)$ \\
\hline \multirow{3}{*}{$\begin{array}{l}\text { Pooled EORTC } 18071 \\
\text { and CheckMate } 238 \\
\text { (stage IIIB/C) }^{\mathrm{b}}\end{array}$} & \multirow{3}{*}{$\begin{array}{l}\text { Nivolumab } \\
\text { vs. } \\
\text { ipilimumab }\end{array}$} & 12 & 74.1 & 61.6 & 12.5 & $7.97(5.13,17.91)$ \\
\hline & & 18 & 70.0 & 54.5 & 15.5 & $6.45(4.39,12.15)$ \\
\hline & & 24 & 66.8 & 51.3 & 15.6 & $6.43(4.34,12.40)$ \\
\hline
\end{tabular}

$C I$ confidence interval, NNT number needed to treat, RFS recurrence-free survival

a Survival rates for treatments and comparators were extracted from the Kaplan-Meier analysis of recurrence-free survival

b In the pooled EORTC 18071 and CheckMate 238 trial data, the number of patients at risk of recurrence at month 0, 12, 18 , and 24 was $278,200,180$, and 131, respectively, for nivolumab; 365, 173, 147, and 133, respectively, for observation; and $644,362,305$, and 247, respectively, for ipilimumab

5.61) for nivolumab versus observation. In other words, four patients would have to be treated with nivolumab compared with observation to avoid one additional recurrence. The NNT continued to decrease numerically at 18 months $(3.55 ; 95 \%$ CI $2.78,4.91)$ and at 24 months $(3.42 ; 95 \%$ CI $2.69,4.67)$.
Compared with ipilimumab at 12 months, the NNT per additional recurrence-free survivor for nivolumab was 7.97 (95\% CI 5.13, 17.91), which continued to decrease numerically at 18 months $(6.45 ; 95 \%$ CI $4.39,12.15)$ and at 24 months $(6.43 ; 95 \%$ CI 4.34, 12.40). 


\section{Costs per Recurrence-Free Life-Month}

The mean drug costs (the sum of drug acquisition and administration costs) per recurrencefree life-month were lower for nivolumab compared with ipilimumab throughout the 24 months of follow-up. For nivolumab, the mean drug costs per recurrence-free life-month decreased from $\$ 13,447$ at 12 months to $\$ 7370$ at 24 months of follow-up, whereas for ipilimumab the mean costs per recurrence-free lifemonth decreased from $\$ 52,734$ to $\$ 33,875$ during the same period (Fig. 2a). The mean medical costs per recurrence-free life-month were the lowest for nivolumab versus observation or ipilimumab through month 12 (\$449 versus $\$ 674$ or $\$ 1531)$ and month 16 (\$383 versus $\$ 808$ or $\$ 1316$ ) (Fig. 2b), driven by lower hospitalization costs (Supplementary Table S2). As follow-up time increased, the mean medical costs per recurrence-free life-month decreased for nivolumab and ipilimumab but increased for observation.

Because of the lower drug and medical costs per recurrence-free life-month, the total costs per recurrence-free life-month were lower for nivolumab versus ipilimumab through month 12 (\$13,895 versus $\$ 54,265$, respectively) and

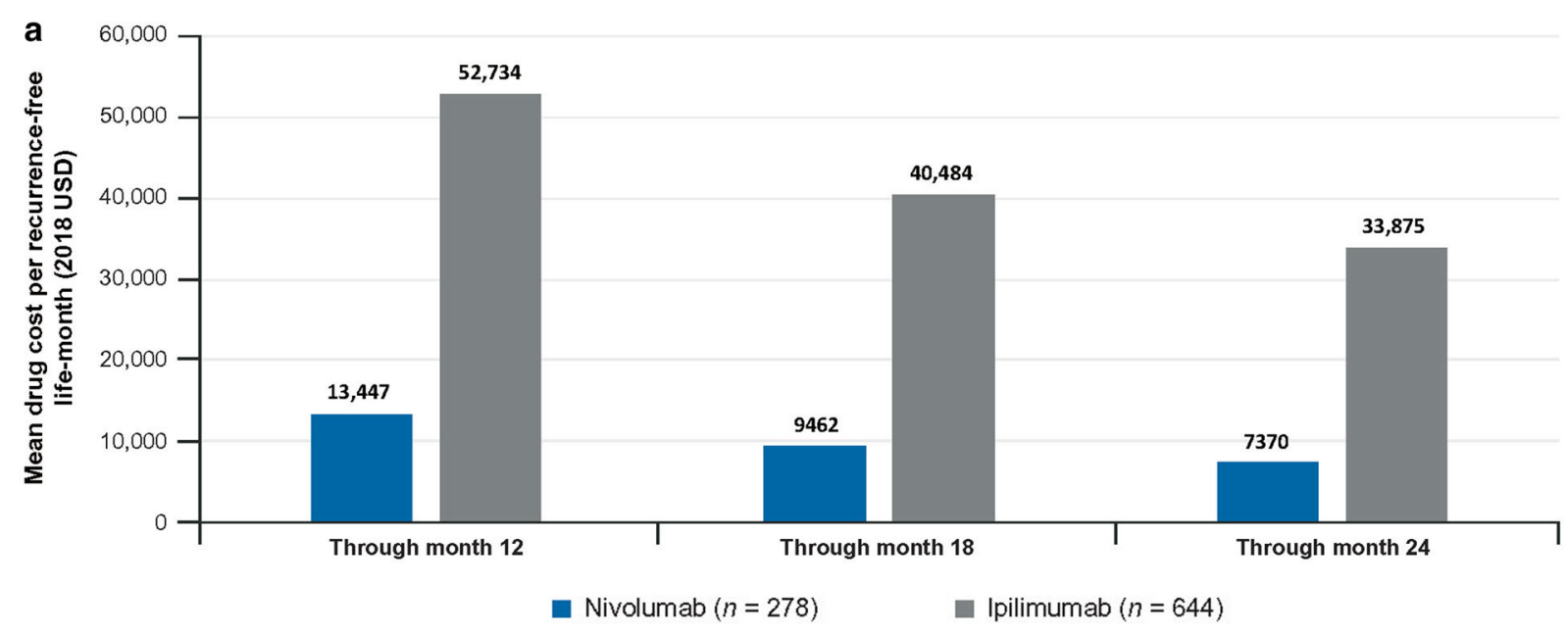

b

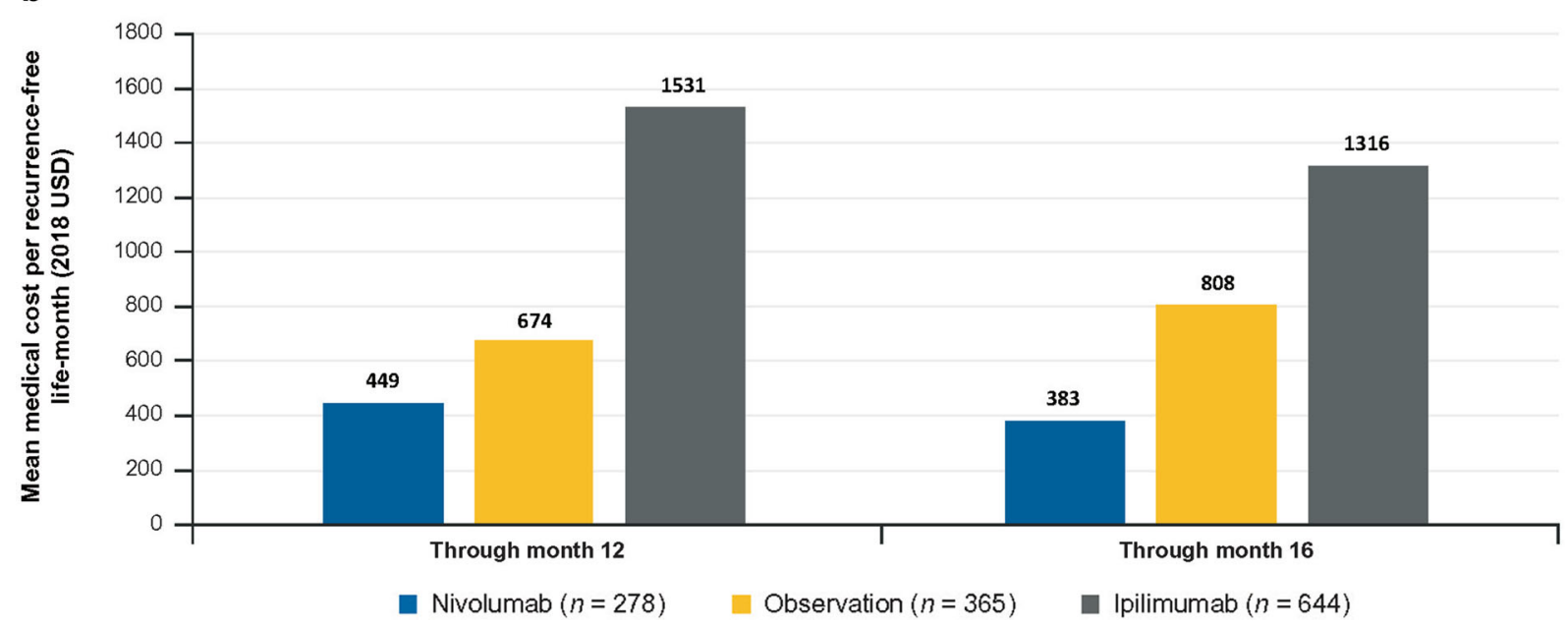

Fig. 2 Mean drug costs per recurrence-free life-month for nivolumab and ipilimumab (a) and mean medical costs per recurrence-free life-month for nivolumab, observation, and ipilimumab (b). Mean drug costs were calculated as the sum of drug acquisition and administration costs. USD United States dollars 
month 16 ( $\$ 10,751$ versus $\$ 44,176$, respectively). In contrast, nivolumab is associated with higher total costs per recurrence-free lifemonth compared with observation through month 12 (\$13,895 versus \$674). A detailed list of drug and medical costs per recurrence-free life-month is available in Supplementary Table S2, along with the duration of recurrencefree life-month throughout each follow-up period.

\section{Costs per Recurrence-Free Survivor}

Similar to the costs per recurrence-free lifemonth, the mean drug costs per recurrence-free survivor were lower for nivolumab (range from $\$ 183,948$ to $\$ 204,088$ ) compared with ipilimumab (range from $\$ 793,779$ to $\$ 1,051,756$ ) throughout the 24 months of follow-up (Table 3). Mean medical costs per recurrencefree survivor were lower for nivolumab versus observation or ipilimumab through month 12 ( $\$ 6136$ versus $\$ 11,282$ or $\$ 23,049$, respectively) and through month 16 (\$7168 versus $\$ 18,403$ or $\$ 27,166$, respectively).

\section{Sensitivity Analysis}

The sensitivity analysis included 906 patients with stage IIIB/C or IV melanoma from the CheckMate 238 trial (453 received nivolumab and 453 received ipilimumab). Results were consistent with the primary analysis, and thus validated the indirect comparison approach of nivolumab versus ipilimumab. The NNT per additional recurrence-free survivor for nivolumab versus ipilimumab was 9.65 (95\% CI 6.06, 23.66) through month $12,7.79$ (95\% CI 5.22, 15.38) through month 18 , and 8.03 (95\% CI $5.30,16.59$ ) through month 24 (Supplementary Table S3).

The mean drug costs per recurrence-free lifemonth were lower for nivolumab versus ipilimumab through month 12 (\$13,202 versus $\$ 53,068$, respectively), through month 18 (\$9389 versus $\$ 38,729$, respectively), and through month 24 (\$7369 versus \$31,115, respectively) (Supplementary Fig. S1a). The mean medical costs per recurrence-free lifemonth were lower for nivolumab versus ipilimumab through month 12 (\$526 versus \$1362,

Table 3 Costs (2018 USD) per recurrence-free survivor among patients with stage IIIB/C melanoma

\begin{tabular}{|c|c|c|c|}
\hline Outcome measures & $\begin{array}{l}\text { Nivolumab } 3 \mathrm{mg} / \mathrm{kg}, \\
n=278\end{array}$ & $\begin{array}{l}\text { Observation, } \\
n=365\end{array}$ & $\begin{array}{l}\text { Ipilimumab } 10 \mathrm{mg} / \mathrm{kg} \text {, } \\
n=644\end{array}$ \\
\hline \multicolumn{4}{|l|}{ Through month 12} \\
\hline Mean total costs per recurrence-free survivor ${ }^{a}$ & 190,085 & 11,282 & 816,827 \\
\hline Mean drug costs per recurrence-free survivor & 183,948 & 0 & 793,779 \\
\hline Mean medical costs per recurrence-free survivor & 6136 & 11,282 & 23,049 \\
\hline \multicolumn{4}{|l|}{ Through month 16} \\
\hline Mean total costs per recurrence-free survivor & 201,437 & 18,403 & 911,903 \\
\hline Mean drug costs per recurrence-free survivor & 194,269 & 0 & 884,738 \\
\hline Mean medical costs per recurrence-free survivor & 7168 & 18,403 & 27,166 \\
\hline \multicolumn{4}{|l|}{ Through month 18} \\
\hline Mean drug costs per recurrence-free survivor & 194,919 & 0 & 947,820 \\
\hline \multicolumn{4}{|l|}{ Through month 24} \\
\hline Mean drug costs per recurrence-free survivor & 204,088 & 0 & $1,051,756$ \\
\hline
\end{tabular}

USD United States dollars

${ }^{a}$ Mean costs per recurrence-free survivor were calculated as mean costs divided by recurrence-free survival rate 
respectively) and month 16 ( $\$ 453$ versus $\$ 1147$, respectively) (Supplementary Fig. S1b). In line with these results, total costs per recurrence-free life-month were lower for nivolumab versus ipilimumab through month 12 ( $\$ 13,728$ versus $\$ 54,430$, respectively) and month $16(\$ 10,711$ versus $\$ 43,114$, respectively). Overall, the cost per recurrence-free life-month and cost per recurrence-free survivor results (Supplementary Table S4) were consistent with the primary analysis. A detailed list of drug and medical costs per recurrence-free life-month is available in Supplementary Table S5.

\section{DISCUSSION}

Adjuvant therapy for cutaneous melanomawhich has historically consisted of interferon (IFN)-based therapy and, more recently, ipilimumab $10 \mathrm{mg} / \mathrm{kg}$ - has long been underutilized for various reasons, including limited survival benefit (IFN-based therapy) and severe adverse events (both IFN-based therapy and ipilimumab) [12, 19]. As a result, patients with melanoma often did not receive any adjuvant treatment after primary tumor resection, despite a high risk of recurrence [27]. However, the recently approved agents have dramatically changed the treatment landscape in the adjuvant setting [28]. As a recently approved adjuvant treatment for melanoma, nivolumab has showed a favorable toxicity profile and efficacy outcomes compared with ipilimumab [22]. Therefore, it is important to understand the comparative efficacy and cost-effectiveness of nivolumab versus observation or ipilimumab.

Given the lack of head-to-head trials comparing nivolumab with observation, we compared the efficacy and costs of nivolumab with that of observation (i.e., no treatment) and ipilimumab using a propensity score approach. Our findings show that nivolumab is more efficacious (as measured by the NNT calculated from RFS) as an adjuvant treatment compared with observation or ipilimumab. Notably, only four patients needed to be treated with nivolumab instead of observation and eight patients needed to be treated with nivolumab instead of ipilimumab to avoid one additional recurrence or death at 12 months. In addition, the NNT decreased to 3.4 for nivolumab versus observation and to 6.4 for nivolumab versus ipilimumab after 24 months, indicating that the clinical benefits of nivolumab increased over time.

The results from this study demonstrated that nivolumab is associated with lower medical costs per recurrence-free life-month compared with observation or ipilimumab in the adjuvant setting. The lower medical costs associated with adjuvant nivolumab were mainly driven by lower hospitalization costs, which may be attributable to fewer recurrences and improved clinical outcomes compared with observation and to a better toxicity profile compared with ipilimumab. The medical cost savings of nivolumab versus observation may extend to 24 months owing to the favorable NNT results of nivolumab versus observation. However, to fully understand the long-term costs of nivolumab versus observation, future analyses with longer follow-up times are warranted to be able to include the cost of any subsequent treatment, as well as the healthcare resource utilization associated with recurrence beyond 16 months, the time point utilized in the current study.

The drug costs (i.e., drug administration and acquisition costs) per recurrence-free life-month for adjuvant nivolumab were also lower than those for adjuvant ipilimumab. Notably, for patients who received nivolumab, drug costs per recurrence-free life-month decreased during the second year of follow-up to nearly half during the first year, suggesting a sustained and long-term economic benefit associated with nivolumab over ipilimumab in the adjuvant setting. A similar trend was observed for the costs to prevent one death or recurrence.

On the other hand, nivolumab is associated with higher total costs per recurrence-free lifemonth when compared with observation through month 12 (\$13,895 vs. $\$ 674)$. This cost difference is primarily due to the drug administration and acquisition costs associated with adjuvant nivolumab, while observation is associated with no drug costs. As shown in this study, the total costs per recurrence-free lifemonth could potentially be offset by the higher 
efficacy and lower medical costs associated with adjuvant nivolumab, with a more pronounced effect over a long-term period. Future analyses with longer follow-up times are warranted to fully assess potential cost offsets beyond 16 months. In addition, it will be important to compare the cost-effectiveness of nivolumab with other adjuvant treatments in melanoma. On the basis of an approximate assessment, the first-year drug acquisition cost of nivolumab was similar to that of pembrolizumab $(\$ 169,898$ based on approved doses) in resected melanoma $[29,30]$. Additionally, the costs associated with anti-PD-1 therapies in resected melanoma were similar to those observed with adjuvant treatment in other cancer types. For example, mean total costs are estimated to be $\$ 217,800$ for 1 year of adjuvant imatinib treatment for gastrointestinal stromal tumors [31]. Future studies comparing the cost per recurrence-free lifemonth between nivolumab and other adjuvant treatments in resected melanoma are needed to better understand the relative cost-effectiveness of nivolumab.

The choice of therapy for patients with melanoma in the adjuvant setting rests on the evaluation of the benefits, risks of both relapse and side effects, and costs associated with each available treatment. The finding that nivolumab is more affordable and has an improved clinical profile compared with ipilimumab provides valuable information for clinical and costeffective decision-making. The availability of nivolumab as a more efficacious treatment option with lower drug and medical costs may increase the number of patients with invasive melanoma who receive adjuvant therapy. Furthermore, although this study did not directly assess adverse events, the CheckMate 238 trial demonstrated that nivolumab had a lower rate of grade $3 / 4$ adverse events compared with ipilimumab ( $14.4 \%$ versus $45.9 \%$, respectively), as well as a lower discontinuation rate because of any adverse event $(9.7 \%$ versus $42.6 \%$, respectively) [22]. The milder toxicity profile of nivolumab further improves its benefit-risk profile in the adjuvant setting.

Although the improvements in NNT (calculated from RFS rates) associated with nivolumab are encouraging, OS is a key outcome for patients and healthcare providers when it comes to adjuvant treatment. In EORTC 18071, ipilimumab demonstrated a significantly higher 7-year OS rate compared with observation (60.0\% versus $51.3 \%$, respectively) [18]. However, OS could not be examined in the current study as the OS data for CheckMate 238 are not yet mature. While RFS is a reasonable surrogate for OS in melanoma [32] and in other types of cancers [33], further analyses comparing the OS associated with nivolumab versus observation or ipilimumab are warranted to fully assess the clinical benefits associated with nivolumab.

This study has several limitations. First, although propensity score weighting can adjust for observed baseline differences between trials, unobserved or unadjustable cross-trial differences may still bias the comparison of outcomes. For example, the maximum treatment period is 3 years in the EORTC 18071 trial [16] and 1 year in the CheckMate 238 trial [22]. This unadjustable cross-trial difference might affect the results of this study beyond 1 year. However, since the actual median number of doses of ipilimumab is four for both trials, the impact of the unadjustable difference in treatment periods between the two trials may be limited. Second, the disease recurrence outcome assessed by an Independent Review Committee (IRC) in the EORTC 18071 trial [16] and the disease recurrence assessed by the study investigators in the CheckMate 238 trial [22] were selected in the present study on the basis of clinical expert opinion. The unmeasurable difference between the two types of outcomes might affect the results; however, the impact should be minimal on the basis of clinical expert opinion. Third, because the patient sample enrolled in the two trials might be different from the melanoma patient population in real-world clinical practice, the results of the current study may not be generalizable to the overall patient population with resected melanoma. In addition, healthcare resource utilization data in CheckMate 238 were collected only up to 128 days after the last dose or treatment discontinuation date [22]. Because the assessment period may vary across patients, the medical costs reported in this study may have been underestimated. Further, the medical service unit costs used in this study 
were obtained from a previous claims database analysis for advanced melanoma [26]. In addition, this study did not analyze the cost-effectiveness of all immune checkpoint inhibitors or targeted therapies in the adjuvant setting. This study provides the best available data since the unit costs were based on real-world evidence in melanoma, although it may overestimate or underestimate the actual costs sustained by patients with resected melanoma. This study did also not estimate the indirect cost savings associated with freedom from relapse, which may contribute to fewer missed days at work and greater economic productivity [34]. Finally, this study focused on drug and medical costs during adjuvant treatment; other costs associated with recurrence, such as subsequent treatment costs and terminal care costs, should be considered in future analyses to provide a more complete cost-effectiveness assessment of adjuvant treatment with nivolumab.

\section{CONCLUSION}

In patients with resected stage IIIB/C melanoma, only four patients need to be treated with nivolumab in order to result in one fewer recurrence event at 12 months. This clinical benefit appeared to strengthen over time, with the NNT being 3.4 at 24 months. In addition, nivolumab was associated with a lower medical cost per recurrence-free life-month and recurrence-free survivor compared with observation, and a lower drug cost per recurrence-free lifemonth and recurrence-free survivor compared with ipilimumab. Although the total cost per recurrence-free life-month is higher for nivolumab compared with observation, it is similar to other checkpoint inhibitors used in resected melanoma. These results point to nivolumab as a promising adjuvant treatment for IIIB/C melanoma after complete resection of the primary tumor. To fully understand the relative costs of nivolumab and observation, future studies with longer follow-up times are warranted to be able to include the impact of subsequent treatment and healthcare resource utilization associated with recurrence beyond the 16 months considered in this study.

\section{ACKNOWLEDGEMENTS}

We thank the participants in the original studies.

Funding. Funding for this research and the journal's Rapid Service and Open Access fees was provided by Bristol-Myers Squibb. All authors had full access to all of the data in this study and take complete responsibility for the integrity of the data and accuracy of the data analysis.

Medical Writing, Editorial, and Other Assistance. We thank Corey Ritchings and Matthew Burke, employees of Bristol-Myers Squibb, for their valuable feedback on the manuscript. Medical writing assistance was provided by Cinzia Metallo, $\mathrm{PhD}$, an employee of Analysis Group, Inc, funded by Bristol-Myers Squibb. Editorial assistance was provided by Kakoli Parai, PhD, and Lilly Ostrovsky at StemScientific, an Ashfield company, funded by Bristol-Myers Squibb.

Authorship. All named authors meet the International Committee of Medical Journal Editors (ICMJE) criteria for authorship for this article, take responsibility for the integrity of the work as a whole, and have given their approval for this version to be published.

Prior Presentation. The data in this article were previously presented in part at the ASCO Annual Meeting 2018.

Disclosures. Morganna Freeman serves on the advisory boards of Bristol-Myers Squibb, Merck, Novartis, and Sanofi Regeneron. Keith A. Betts is an employee of Analysis Group, Inc., which has received consultancy fees from Bristol-Myers Squibb to conduct this study. Shan Jiang was an employee of Bristol-Myers Squibb at the time of this study. Ella X. Du is an employee of Analysis Group, Inc., which has received consultancy fees from Bristol-Myers Squibb to conduct this study. Komal GupteSingh is an employee of Bristol-Myers Squibb. Yichen $\mathrm{Lu}$ is an employee of Analysis Group, Inc., which has received consultancy fees from 
Bristol-Myers Squibb to conduct this study. Sumati Rao is an employee of Bristol-Myers Squibb. Alexander N. Shoushtari serves on the advisory boards of Bristol-Myers Squibb, Castle Biosciences, and Immunocore; and has received institutional research support from AstraZeneca, Bristol-Myers Squibb, Immunocore, Polaris, and Xcovery.

Compliance with Ethics Guidelines. Original data from the EORTC 18071 and the CheckMate 238 trials were collected within an ethical framework. These clinical trials were performed in accordance with the Helsinki declaration, had approval from respective institutional review boards, and had obtained informed consent from all participants.

Data Availability. BMS policy on data sharing may be found at https://www.bms.com/ researchers-and-partners/independent-research/ data-sharingrequest-process.html.

Open Access. This article is distributed under the terms of the Creative Commons Attribution-NonCommercial 4.0 International License (http://creativecommons.org/licenses/ by-nc/4.0/), which permits any noncommercial use, distribution, and reproduction in any medium, provided you give appropriate credit to the original author(s) and the source, provide a link to the Creative Commons license, and indicate if changes were made.

\section{REFERENCES}

1. AIM At Melanoma Foundation. Melanoma stats, facts, and figures. https://www.aimatmelanoma. org/about-melanoma/melanoma-stats-facts-andfigures/. Cited August 2018.

2. National Cancer Institute. Cancer stat facts: melanoma of the skin. https://seer.cancer.gov/statfacts/ html/melan.html. Cited August 2018.

3. American Cancer Society. Key statistics for melanoma skin cancer. https://www.cancer.org/cancer/ melanoma-skin-cancer/about/key-statistics.html. Cited June 2019.
4. Cancer.Net. Melanoma: statistics. https://www. cancer.net/cancer-types/melanoma/statistics. Cited August 2018.

5. Melanoma Research Foundation. Metastatic melanoma. https://www.melanoma.org/understand-mela noma/what-is-melanoma/metastatic-melanoma. Cited August 2018.

6. AIM At Melanoma Foundation. Recurrence of Melanoma. https://www.aimatmelanoma.org/after-treat ment/recurrance-of-melanoma/. Cited August 2018.

7. Medscape. Malignant melanoma treatment \& management. https://emedicine.medscape.com/ article/280245-treatment. Cited August 2018.

8. American Cancer Society. Treatment of melanoma skin cancer, by stage. https://www.cancer.org/ cancer/melanoma-skin-cancer/treating/by-stage. html. Cited August 2018.

9. Coit DG, Thompson JA, Albertini MR, et al. Cutaneous melanoma, version 2.2019, NCCN Clinical Practice Guidelines in Oncology. J Natl Compr Canc Netw. 2019;17:367-402.

10. UpToDate. Adjuvant therapy for cutaneous melanoma. https://www.uptodate.com/contents/adjuvant -therapy-for-cutaneous-melanoma. Cited August 2018.

11. Romano E, Scordo M, Dusza SW, Coit DG, Chapman PB. Site and timing of first relapse in stage III melanoma patients: implications for follow-up guidelines. J Clin Oncol. 2010;28:3042-7.

12. Bello DM, Ariyan CE. Adjuvant therapy in the treatment of melanoma. Ann Surg Oncol. 2018;25:1807-13.

13. Napolitano S, Brancaccio G, Argenziano G, et al. It is finally time for adjuvant therapy in melanoma. Cancer Treat Rev. 2018;69:101-11.

14. Zhang Y, Le TK, Shaw JW, Kotapati S. Retrospective analysis of drug utilization, health care resource use, and costs associated with IFN therapy for adjuvant treatment of malignant melanoma. Clinicoecon Outcomes Res. 2015;7:397-407.

15. National Comprehensive Cancer Network (NCCN). NCCN clinical practice guidelines: Melanoma. https://www.nccn.org/store/login/login.aspx?Return URL=https://www.nccn.org/professionals/physici an_gls/PDF/melanoma.pdf. Cited November 2018.

16. Eggermont AM, Chiarion-Sileni V, Grob JJ, et al. Prolonged survival in stage III melanoma with ipilimumab adjuvant therapy. $\mathrm{N}$ Engl J Med. 2016;375:1845-55. 
17. Eggermont AM, Chiarion-Sileni V, Grob JJ, et al. Adjuvant ipilimumab versus placebo after complete resection of high-risk stage III melanoma (EORTC 18071): a randomised, double-blind, phase 3 trial. Lancet Oncol. 2015;16:522-30.

18. Eggermont AM, Chiarion-Sileni V, Grob JJ, et al. Ipilimumab versus placebo after complete resection of stage III melanoma: long-term follow-up results of the EORTC 18071 double-blind phase 3 randomized trial. Presented at American Society of Clinical Oncology 2019; abstract \#2512.

19. Goldstein DA. Adjuvant ipilimumab for melanoma-the $\$ 1.8$ million per patient regimen. JAMA Oncology. 2017;3:1628-9.

20. Food and Drug Administration (FDA). FDA grants regular approval to nivolumab for adjuvant treatment of melanoma. https://www.fda.gov/drugs/ informationondrugs/approveddrugs/ucm590004. htm. Cited August 2018.

21. Weber J, Mandala M, Del Vecchio M, et al. Adjuvant therapy with nivolumab (NIVO) versus ipilimumab (IPI) after complete resection of stage III/IV melanoma: updated results from a phase III trial (CheckMate 238). Presented at American Society of Clinical Oncology 2018; abstract \#9502.

22. Weber J, Mandala M, Del Vecchio M, et al. Adjuvant nivolumab versus ipilimumab in resected stage III or IV melanoma. N Engl J Med. 2017;377:1824-35.

23. Altman DG, Andersen PK. Calculating the number needed to treat for trials where the outcome is time to an event. BMJ. 1999;319:1492-5.

24. Analysource. Drug pricing database. https://www. analysource.com/. Cited January 2018.

25. Centers for Medicare \& Medicaid Services (CMS). Physician fee schedule. https://www.cms.gov/ Medicare/Medicare-Fee-for-Service-Payment/ PhysicianFeeSched/. Cited June 2018.
26. Potluri R, Okoro T, Bhandari $\mathrm{H}$, et al. Health care cost comparison analysis of nivolumab + ipilimumab regimen and nivolumab monotherapy versus established advanced melanoma therapies utilizing clinical trial and real-world data. Value Health. 2016;19:A144.

27. Tarhini A, Gupte-Singh K, You M, et al. Abstract 3641: use of adjuvant interferon alfa-2b or ipilimumab $10 \mathrm{mg} / \mathrm{kg}$ for high-risk patients with melanoma, and associated adverse events and duration of therapy. Cancer Res. 2018;78:3641.

28. Testori AAE, Ribero S, Indini A, et al. Adjuvant treatment of melanoma: recent developments and future perspectives. Am J Clin Dermatol. 2019. https://doi.org/10.1007/s40257-019-00456-4.

29. IBM Micromedex ${ }^{\circledR}$ RED BOOK. http://www. micromedexsolutions.com. Cited March 2019.

30. Eggermont AMM, Blank CU, Mandala M, et al. Adjuvant pembrolizumab versus placebo in resected stage III melanoma. N Engl J Med. 2018;378:1789-801.

31. Sanon M, Taylor DC, Parthan A, et al. Cost-effectiveness of 3-years of adjuvant imatinib in gastrointestinal stromal tumors (GIST) in the United States. J Med Econ. 2013;16:150-9.

32. Suciu S, Eggermont AMM, Lorigan P, et al. Relapsefree survival as a surrogate for overall survival in the evaluation of stage II-III melanoma adjuvant therapy. J Natl Cancer Inst. 2018;110:87-96.

33. Araujo RLC, Herman P, Riechelmann RP. Recurrence-free survival as a putative surrogate for overall survival in phase III trials of curative-intent treatment of colorectal liver metastases: systematic review. World J Clin Oncol. 2017;8:266-72.

34. Buja A, Sartor G, Scioni M, et al. Estimation of direct melanoma-related costs by disease stage and by phase of diagnosis and treatment according to clinical guidelines. Acta Derm Venereol. 2018;98:218-24. 